\title{
Cerebellar lncRNA Expression Profile Analysis of SCA3/MJD Mice
}

\author{
Zhe Long, ${ }^{1,2}$ Tianjiao Li, ${ }^{1}$ Zhao Chen, ${ }^{1}$ Yun Peng, ${ }^{1}$ Chunrong Wang, ${ }^{1}$ Xiaocan Hou, ${ }^{1}$ \\ Hongyu Yuan, ${ }^{1}$ Puzhi Wang, ${ }^{1}$ Yue Xie, ${ }^{1}$ Lang He, ${ }^{1}$ Xin Zhou, ${ }^{1}$ Huirong Peng, ${ }^{1}$ Rong Qiu, ${ }^{3}$ \\ Kun Xia, ${ }^{4}$ Beisha Tang $\mathbb{D}^{1,4,5,6,7,8,9}$ and Hong Jiang $\mathbb{D}^{1,4,6,10}$ \\ ${ }^{1}$ Department of Neurology, Xiangya Hospital, Central South University, Changsha, Hunan 410008, China \\ ${ }^{2}$ Sydney Medical School and the Brain \& Mind Institute, The University of Sydney, 94 Mallett St, Camperdown, NSW 2050, Australia \\ ${ }^{3}$ School of Information Science and Engineering, Central South University, Changsha, Hunan 410083, China \\ ${ }^{4}$ Laboratory of Medical Genetics, Central South University, Changsha, Hunan 410078, China \\ ${ }^{5}$ National Clinical Research Center for Geriatric Diseases, Xiangya Hospital, Central South University, Changsha, \\ Hunan 410008, China \\ ${ }^{6}$ Key Laboratory of Hunan Province in Neurodegenerative Disorders, Central South University, Changsha, Hunan 410008, China \\ ${ }^{7}$ Parkinson's Disease Center of Beijing Institute for Brain Disorders, Beijing 100069, China \\ ${ }^{8}$ Collaborative Innovation Center for Brain Science, Shanghai 200032, China \\ ${ }^{9}$ Collaborative Innovation Center for Genetics and Development, Shanghai 200433, China \\ ${ }^{10}$ Xinjiang Medical University, Xinjiang 830011, China
}

Correspondence should be addressed to Hong Jiang; jianghong73868@126.com

Received 1 March 2018; Revised 9 April 2018; Accepted 27 May 2018; Published 25 June 2018

Academic Editor: Elena Pasyukova

Copyright ( 2018 Zhe Long et al. This is an open access article distributed under the Creative Commons Attribution License, which permits unrestricted use, distribution, and reproduction in any medium, provided the original work is properly cited.

Spinocerebellar ataxia type 3 (SCA3) or Machado-Joseph disease (MJD) is the most common autosomal dominant spinocerebellar ataxia in China with highly clinical heterogeneity, such as progressive cerebellar ataxia, dysarthria, pyramidal signs, external ophthalmoplegia, dysphagia, and distal muscle atrophy. It is caused by the abnormal expansion of CAG repeats in a coding region of ATXN3. However, by focusing on the ATXN3 itself cannot fully explain the heterogeneous clinical features of SCA3/ MJD. With the discovery of the increasing number of long noncoding RNAs (lncRNAs) that are believed to be involved in spinocerebellar ataxia type 8 (SCA8) and Huntington disease (HD), we wonder whether the lncRNAs are differentially expressed in the SCA3/MJD patients compared to the nonpatients. As the first step, we used lncRNA-Seq to investigate differential expression of the lncRNAs in the SCA3/MJD mice. Two known lncRNAs, n297609 and n297477, and a novel lncRNA TCONS_00072962 have been identified in SCA3/MJD mice with abnormal expression. The first discovery of the novel lncRNA TCONS_00072962 enriched the lncRNA expression profile in the SCA3/MJD mouse model.

\section{Introduction}

PolyQ diseases is a group of disorders caused by CAG repeat expansions within the, respectively, responsible genes, including Huntington disease (HD), dentatorubral-pallidoluysian atrophy (DRPLA), spinocerebellar ataxias (SCA1, SCA2, SCA3/Machado-Joseph disease, SCA6, SCA7, and SCA17) [1-3], and the recently discovered Huntington disease-like 2 (HDL2) $[4,5]$. Among these, the SCA3/MJD is an autosomal dominantly inherited disorder with high clinical heterogeneity, such as progressive cerebellar ataxia, dysarthria, pyramidal signs, external ophthalmoplegia, dysphagia, and distal muscle atrophy, with wide range of age of onset (AO) from 4 to 75 years old $[6,7]$.

The SCA3/MJD, the most common case, accounts for $62.64 \%$ of autosomal dominant spinocerebellar ataxia in China [8]. The abnormal expansion of CAG in the causative gene ATXN3 coding region causes SCA3/MJD. Healthy individuals usually have 12-40 CAG repeats, while SCA3/MJD patients over 51 repeats $[9,10]$. The abnormally translated 
polyQ tract leads to a conformational change in ATXN3, resulting in alternations of protein properties, including stability, subcellular location, and easier aggregation [11]. These alternations further lead to loss or gain of function and cause pathogenic effects. To explain the toxic effects, several hypotheses of pathogenic mechanisms, not mutually exclusive, have been presented, including aggregate formation $[1,11-13]$, disturbance of cellular protein and $\mathrm{Ca} 2+$ homeostasis [13-15], dysregulation of transcription [15, 16], axonal transport deficits $[17,18]$, impairment of mitochondrial function $[15,19,20]$, and abnormal neuronal signalling [11].

Long noncoding RNA (lncRNA) is defined the nontranslatable RNA with the length of 200 nucleotides or above. The lncRNAs used to be regarded as the transcriptional "noise," the products of RNA polymerase II transcription, and did not have the biological function. However, the emerging evidence has proved their significant roles in the regulation of gene transcription, posttranscriptional regulation, and epigenetic regulation [21, 22]. Previous studies suggested that lncRNAs regulate the gene expression and transcriptional processes by several different functional mechanisms. Some show function as transcriptional regulation in cis or trains, some as an organization of nuclear domains, and others as regulation of proteins or RNA molecules. All the evidence indicated that lncRNAs have great potential to impact physiological and pathological processes. Furthermore, it has been found that some transcripts of lncRNA encode small proteins [23], making the noncoding inappropriate any longer to name this class of RNA.

In recent years, accumulating studies have found that lncRNAs are associated with neurodegenerative diseases. Spinocerebellar ataxia type 8 (SCA8), a kind of slowly progressive ataxia, is caused by the abnormal expansion of (CTG)n within the responsible gene ATXN8. A study proposes that the pathogenesis of SCA8 involves both protein and RNA gain-of-function mechanisms. (CTG)n-expanded ATXN8 encodes a pathogenic protein, and the antisense strand encodes CUG-enriched lncRNA ATXN8OS which is deposited in the nucleus and activates alternative splicing, resulting in an alternation of the expression of GABA-A transport factor 4 (GAT4/Gabt4) and finally loss of the GABAergic inhibition [24].

In a separate study, the expression of lncRNA was compared between the normal brain tissue and the brain tissue of patients with Huntington disease. A total of 35 upregulated and 146 downregulated IncRNA molecules were identified, and NEAT1 was selected by Bioinformatics. Based on the cell-level experiments, it was found that overexpression of NEAT1 was significantly resistant to $\mathrm{H}_{2} \mathrm{O}_{2}$-induced cellular damage, providing a new potential strategy for clinical treatment of the Huntington's disease [25].

To further explore the pathogenesis of SC3/MJD at RNA level, the lncRNAs specifically expressed in SCA3/MJD mice were investigated in this study.

\section{Materials and Methods}

2.1. SCA3/MJD Mice. SCA3/MJD mouse model (B6; CBA-Tg (ATXN3*) 84.2Cce/IbezJ; ID: 012075) from Jackson
Laboratory was used, and the second generation was used in this study. The CAG repeats in the first generation mice are 84, and the ATXN3 gene is widely expressed in various organs of the body, including the cerebellum, cerebral cortex, heart, lung, spleen, liver, and skeletal muscle [26, 27]. The SCA3/MJD adult mice (32 weeks old) of the second generation, in which carrying ATXN3 positive rate is about $50 \%$, and comparable age, number, and weight wild-type mice were used for experimental analysis. The study was approved by the Ethics Committee in Xiangya Hospital of Central South University.

2.2. Validation of Genotype of SCA3/MJD Mice. Validation of genotype was conducted in the second generation. Polymerase chain reaction (PCR), agarose gel electrophoresis, and capillary electrophoresis sequencing were used for genotype validation. Genomic DNA was extracted from mice tails. CAG repeats were amplified using a pair of primers $5^{\prime}$-CCAGTGACTACTTTGATTCG-3' (forward) $5^{\prime}$-TGGCCTTTCACATGGATGTGAA- $3^{\prime}$ (reverse). The amplification reactions contained $1 \mu \mathrm{L}$ genomic DNA (50 ng/ $\mu \mathrm{L}$ ), $0.2 \mu \mathrm{L}$ rTaq DNA polymerase (Takara, Japan), $0.2 \mu \mathrm{L}$ dNTPs, $0.2 \mu \mathrm{L}$ of each primer $(100 \mathrm{ng} / \mu \mathrm{L}), 7.2 \mu \mathrm{L}$ sterile water, and $1.0 \mu \mathrm{L} \mathrm{10x}$ buffer (TaKaRa, Japan), for a total of $10 \mu \mathrm{L}$. The amplification was performed in Mastercyclers (Eppendorf AG, 22331 Hamburg, Germany) under the following conditions: initial denaturation at $95.0^{\circ} \mathrm{C}$ for 5 minutes, followed by 38 cycles of $95.0^{\circ} \mathrm{C}$ for 30 seconds, $59.0^{\circ} \mathrm{C}$ for 30 seconds, and $72.0^{\circ} \mathrm{C}$ for 30 seconds. PCR products were detected by $1 \%$ agarose gel electrophoresis $(120 \mathrm{v}$, $30 \mathrm{~min}$ ), and the results of PCR amplification were observed on imaging system after 15 minutes of ethidium bromide (EB) staining. Capillary electrophoresis sequencing was used for testing the repeats number of (CAG)n and performed on ABI 3730XL DNA Analyzer (Applied Biosystems, Foster City, CA, USA).

2.3. Validation of Phenotype of SCA3/MJD Mice. The phenotype was validated using the footprint and rotating tests. For footprint pattern analysis, the hind paws of mice were painted with black ink and the forepaws were painted with red ink. The mice walked along a narrow corridor paved with white paper. Pretraining was conducted for one week before the formal test. Mice were tested three times with 5-minute intervals. Stride length, hind paw width, front paw width, and front/hind footprint overlap were measured. For rotation, mice were placed on a rotating rod and must maintain its balance. The interval from the start of the rod rotating to the mice falling from the rotating rod was recorded. Mice were tested on separate trials at fixed speeds including $10 \mathrm{r} /$ $\mathrm{min}$ and $20 \mathrm{r} / \mathrm{min}$.

2.4. IncRNA-Seq. IncRNA-Seq, a high-throughput sequencing, was performed in BGI. After extracting the total RNA from mice cerebellum (three SCA3/MJD mice versus three wild-type mice), mRNA and noncoding RNAs are enriched by removing rRNA from the total RNA. By using the fragmentation buffer, the mRNAs and noncoding RNAs are fragmented into short fragments (about 200 500 nt), then 
TABLE 1: The results of rotation test.

\begin{tabular}{lcccc}
\hline $\begin{array}{l}\text { Rotation } \\
\text { speed }\end{array}$ & $\begin{array}{c}\text { Time } \\
\text { (seconds) }\end{array}$ & $\begin{array}{c}\text { Wild-type } \\
\text { mice }(n=6)\end{array}$ & $\begin{array}{c}\text { SCA3/MJD } \\
\text { mice }(n=6)\end{array}$ & $\begin{array}{c}p \\
\text { value }\end{array}$ \\
\hline $10 \mathrm{r} / \mathrm{min}$ & Mean \pm SD & $250.067 \pm 9.487$ & $104.400 \pm 8.902$ & 0.023 \\
$20 \mathrm{r} / \mathrm{min}$ & Mean \pm SD & $196.467 \pm 8.126$ & $34.600 \pm 6.710$ & 0.002 \\
\hline
\end{tabular}

The wild-type mice performed much better than the SCA3/MJD mice in the rotation test.

the first-strand cDNA is synthesized by random hexamerprimer using the fragments as templates, and dTTP is substituted by dUTP during the synthesis of the second strand. Short fragments are purified and resolved with EB buffer for end reparation and single nucleotide A (adenine) addition. After that, the short fragments are connected with adapters, and then the second strand is degraded using UNG (uracil-N-glycosylase) finally [28]. After agarose gel electrophoresis, the suitable fragments are selected for the PCR amplification as templates. During the QC steps, Agilent 2100 Bioanalyzer and ABI StepOnePlus real-time PCR system are used in quantification and qualification of the sample library. At last, the library could be sequenced using Illumina HiSeqTM 2000 or other sequencers when necessary. Differential expression analysis for both predicted novel lncRNA and lncRNA from the database has proceeded. It was compared between SCA3/MJD and wild-type mice (one by one, grouped randomly) through the Cuffdiff software to calculate the FPKM value of the gene or transcript in both samples and to detect the presence of differential expression. Also, group differential expression analysis was also performed by NOIseq method.

2.5. Quantitative Real-Time PCR. The total RNA from mice cerebellum (six SCA3/MJD mice versus six wild-type mice) was reversely transcribed to cDNA with a kit (Thermo Scientific, RevertAid First Strand cDNA \#K1622). The 44 most differentially expressed lncRNAs were screened for further validation by qRT-PCR assays (Maxima SYBR Green qPCR Master Mix, CFX96, Bio-Rad, USA). $\beta$-Actin was used as an internal reference in the qRT-PCR analyses. The primers (see Table S1 in the Supplementary Material) were designed using Primer3 (http://bioinfo.ut.ee/primer3-0.4.0/). qRT-PCR assay was performed in triplicate in a volume of $20 \mu \mathrm{L}$ containing $1 \mu \mathrm{L}$ of cDNA. The relative expression level of IncRNA was calculated using the $2^{-\Delta \Delta \mathrm{Ct}}$ method and $\mathrm{Ct}>35$ were excluded. A Wilcoxon rank sum test was used for statistical analyses, and $p<0.05$ was considered statistical significance.

2.6. Bioinformatics Analysis. We conducted some biological analysis of the differentially expressed lncRNAs by search and comparison of databases such as NONCODE v5 (http://www.noncode.org/index.php), FANTOM5 (http:// fantom.gsc.riken.jp/5/), STRING v.10.0 (http://version10. string-db.org/), and Gene Ontology Consortium (http:// www.geneontology.org/) to predict the location, distribution, and function of these lncRNAs.

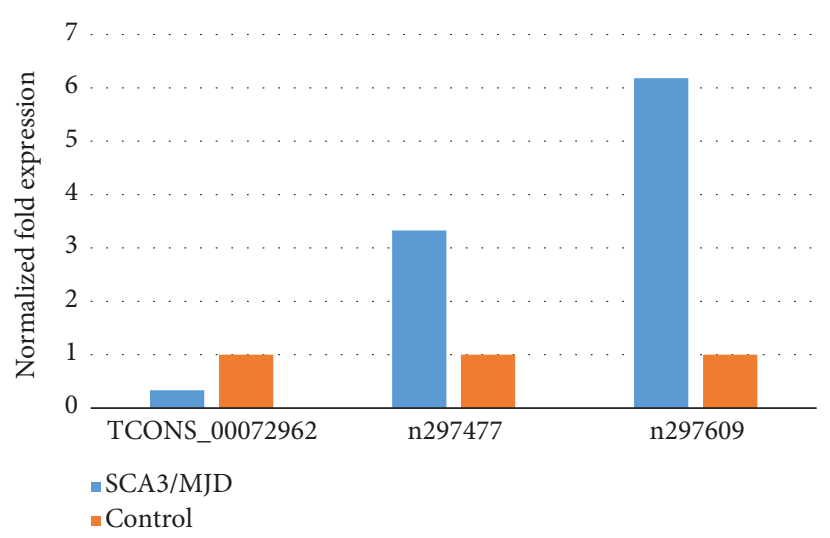

FIgURE 1: The expression level of the three lncRNAs. The expression level of three lncRNAs was different between wild-type and SCA3/ MJD mice with statistical significance. The two known lncRNAs, n297477 and n297609, were upregulated in the cerebellum of SCA3/MJD mice. The expression level was increased by 3.329 times $(p=0.016)$ and 6.182 times $(p=0.041)$, respectively. The novel lncRNA TCONS_00072962 was downregulated $(p=0.036)$ in the cerebellum of SCA3/MJD mice, which was nearly one-third of that in wild-type mice.

\section{Results}

3.1. Genotyping and Phenotyping of the SCA3/MJD Mice. The SCA3/MJD mice that carry the ATXN3 were genotyped and phenotyped. As a result, the ATXN3 carrying rate was about $50 \%$ in the second generation of SCA3/MJD mice, by Jackson Laboratory's report. The number of CAG repeats was 84 or 83 in the second generation SCA3/MJD mice. In rotation test, the interval of mice keeping balance on the rotating rod was significantly different between the SCA3/MJD and wild-type mice no matter the rotation speed was $10 \mathrm{r} / \mathrm{min}$ or $20 \mathrm{r} / \mathrm{min}$ (see Table 1), indicating that the balance and motor abilities of the SCA3/MJD mice were much worse relative to the control mice. Morphologically, wider hind paw width was evident in SCA3/MJD mice.

Abnormal gait was observed by analyzing the footprint pattern. In contrast to the control mice's straight line with regular alternating gait, the SCA3/MJD mice showed unstable movements in a way that weaved from side to side when walked along the narrow corridor. Altogether suggest that the SCA3/MJD mice have similar clinical manifestations with the SCA3/MJD patients.

3.2. IncRNA-Seq. By using RNA-seq, 10,443 of novel and 13,395 of known lncRNAs were detected in 3 of the SCA3/ MJD and 3 of the WT mice. One by one differential expression analysis showed total 2964 upregulated and 4376 downregulated IncRNAs, respectively, in the three experimental groups. More specifically, there were 745, 776, and 1250 of the upregulated lncRNAs in three groups, in contrast to the significantly increased numbers of 1285, 1065, and 1600, the downregulated lncRNAs, respectively, in three groups. Further group differential expression analysis found 193 of the upregulated and 467 of the downregulated lncRNAs in the SCA3/MJD mice. The IncRNAs with differential 
TABLE 2: The summary of genetic association.

\begin{tabular}{lcc}
\hline Gene name & Associated gene name & Proteins of associated gene \\
\hline & VCP & Valosin containing protein \\
UBC & Ubiquitin C \\
KCTD10 & RAD23 homolog A \\
RAD23A & RAD23 homolog B \\
RAD23B & Parkinson protein 2 \\
PARK2 & Ubiquitin specific peptidase 13 \\
& USP13 & Ubiquitination factor E4B \\
UBE4B & STUB1 & STIP1 homology and U-box containing protein 1 \\
& SERPINC1 & Serpin peptidase inhibitor, clade C (antithrombin), member 1 \\
\hline PSMD4 & Proteasome (prosome, macropain) 26S subunit, non-ATPase 4 \\
HSP90AA1 & Heat shock protein 90 kDa alpha (cytosolic), class A member 1 \\
HBC & Hepatocyte growth factor-regulated tyrosine kinase substrate \\
& PSMC2 & Proteasome (prosome, macropain) 26S subunit, ATPase 2 \\
& TSG101 & Tumor susceptibility gene 101 \\
& PSMD14 & Ubiquitin-conjugating enzyme E2D 2 \\
CUL1 & Proteasome (prosome, macropain) 26S subunit, non-ATPase 14 \\
& Cullin 1
\end{tabular}

expression identified by the one by one differential expression analysis in two or three groups were chosen for further validation. The $\operatorname{lncRNAs}$ were sorted based on their FPKM and $p$ values in the group differential expression analysis. For both differential expression analyses, a total of $44 \operatorname{lncRNAs}$ were chosen for further experiments because they were statistically significant.

3.3. $q R T-P C R$. IncRNA number TCONS_00031478 was excluded due to $\mathrm{Ct}>35$. In the rest 43 of the chosen lncRNAs, 3 of the lncRNAs were validated to be differential expression between SCA3/MJD and WT mice cerebellum. It turned out that two of the three are the known lncRNAs number $\mathrm{n} 297477(p=0.016)$ and number n297609 $(p=0.041)$ (NONCODE v5.0: http://www.noncode.org/), and the remaining one belonged to a novel lncRNA (number TCONS_00072962 $)(p=0.036) .2^{-\triangle \triangle C t}$ method was used for calculating the relative expression level of each lncRNA. When compared with wild-type mice, the two known lncRNAs, n297477 and n297609, were upregulated by 3.329-fold and 6.182-fold, respectively, in the cerebellum of SCA3/MJD mice (see Figure 1), while the expression level of novel lncRNA TCONS_00072962 was downregulated in the cerebellum of SCA3/MJD mice, which was nearly onethird of that in control mice.

\section{Discussion}

In this study, 2964 upregulated lncRNAs and 4376 downregulated lncRNAs were identified specifically in the SCA3/MJD mice using lncRNA-Seq analysis. Additionally, differentially expressed three lncRNAs, including one novel lncRNA and two known lncRNAs, were further characterized.
The lncRNA n297477 is transcribed from the antisense strand of the chr11: 6270375-6271530, which is highly expressed in mouse heart, hippocampus, liver, lung, spleen, and thymus. According to the records in database FANTOM5, n297477 is considered one of the transcripts of the TMED4 gene whose promoter is located in the sense strand at position 142-392 (chr11: 6270517-6270767) and TATA box starts at 377 (chr11: 6270712) (http://wwwbimas.cit.nih.gov/molbio/proscan/). According to the database STRING v.10.0, Tmed4 and ubiquitin C may be functional partners to each other, whereas the ubiquitin $C$ might be a functional partner of ataxin 3 encoded by the SCA3/MJD pathogenic gene ATXN3 (Table 2; http:// version10.string-db.org/cgi/network.pl?all_channels_on=1\& block_structure_pics_in_bubbles $=0$ \&direct_neighbor $=1 \&$ hi de_disconnected_nodes $=0$ \&hide_node_labels $=0$ \&network_ display_mode $=$ svg\&network_flavor=evidence\&targetmode= proteins\&identifier $=9606$. ENSP00000376965; http://version 10.string-db.org/cgi/network.pl?all_channels_on=1\&block_ structure_pics_in_bubbles $=0 \&$ direct_neighbor $=1 \&$ hide_dis connected_nodes $=0$ \&hide_node_labels $=0$ \&network_display_ mode $=$ svg\&network_flavor $=$ evidence\&targetmode $=$ proteins \&identifier=9606.ENSP00000404042).

Given that the ubiquitin-proteasome system (UPS) is involved in the pathogenesis of SCA3/MJD [29], it is plausible to speculate that lncRNA may participate in gene expression regulation when it is located close to the transcription start site of the promoter region. Since the lncRNA n297477 and TMED4 gene starting site are separated by $16 \mathrm{bp}$ in the middle, and the gene promoter region is located in the lncRNA coding region, it is highly possible that the n297477 participates in TMED4 expression regulation. We assume that the altered expression of 
the TMED4 affects its interaction with UBC, which affects the efficiency of ubiquitin $\mathrm{C}$ in the ubiquitin-proteasome system. Thus, the lncRNA n297477 may be involved in the regulation of ataxin-3 protein degradation by regulating UPS. The elevated expression of n297477 in the SCA3/MJD mice may serve as a response to the abnormal degradation of toxic ataxin-3.

The lncRNA n297609 is transcribed from the antisense strand of the chr9: 44047488-43856016, which is highly expressed in mouse heart, hippocampus, liver, lung, spleen, and thymus. According to the records in database FANTOM5, n297609 is considered one of the transcripts of the THY1 gene which is highly homologous to humans. The lncRNA n297609 mainly participates in the protein phosphorylation (GO: 0006468), cell adhesion (GO: 0007155), cellular response to heat (GO: 0034605), positive regulation of transcription (GO: 0045893), and negative regulation of cell migration (GO: 0030336). Previous studies pointed out that protein casein kinase 2- (CK2-) dependent phosphorylation can control the stability, nuclear localization, and aggregation of ataxin-3 [30]. Thus, it is logical to assume that the lncRNA n297609 may be involved in biological processes, such as protein phosphorylation, and protein casein kinase 2- (CK2-) dependent phosphorylation plays a crucial role in SCA3/MJD pathophysiology [30]. However, this assumption requires further validation.

Moreover, we also found a novel lncRNA TCONS 00072962 with the genomic location at chr7:119737479119737966 , contributing to the expansion of lncRNA expression profiles of the mouse.

\section{Conclusions}

In summary, we used lncRNA-Seq to profile cerebellar expression in SCA3/MJD mice and identified three potential lncRNAs significantly associated with the disease. These identified lncRNAs will be beneficial for the further understanding of cerebellum gene coexpression network correlating with disease progression. Furthermore, investigation on the IncRNA-associated neuroprotective factors remains to be necessary for the elucidation of the therapeutic target implication.

\section{Data Availability}

The location of all lncRNAs is from the mm10 database.

\section{Conflicts of Interest}

The authors declare no conflict of interest.

\section{Authors' Contributions}

Zhe Long and Tianjiao Li contributed equally to this work.

\section{Acknowledgments}

This study was supported by the National Natural Science Foundation of China (nos. 81471156 and 81771231 to Hong Jiang; nos. 81430023 and 81130021 to Beisha Tang; no.
31401135 to Rong Qiu), National Key Research and Development Program of China (nos. 2016YFC0901504 and 2016YFC0905100 to Hong Jiang; no. 2016YFC1306000 to Beisha Tang), Clinical Research Funds of Xiangya Hospital (no. 2014L03 to Hong Jiang), the Clinical and Rehabilitation Fund of Peking University Weiming Biotech Group (no. xywm2015I10 to Hong Jiang), Youth Foundation of Xiangya Hospital (no. 2017Q03 to Zhao Chen), and Independent Exploration and Innovation Project of Graduate Student of Central South University (no. 1053320170177 to Tianjiao Li). The authors want to give the sincerest thanks to all those who helped in the study.

\section{Supplementary Materials}

Table S1: the supplementary material included 44 lncRNA locations, dysregulation (upregulated/downregulated), and primers used for qPCR amplification. (Supplementary Materials)

\section{References}

[1] A. Ashkenazi, C. F. Bento, T. Ricketts et al., "Polyglutamine tracts regulate beclin 1-dependent autophagy," Nature, vol. 545, no. 7652, pp. 108-111, 2017.

[2] S. Esteves, S. Duarte-Silva, and P. Maciel, "Discovery of therapeutic approaches for polyglutamine diseases: a summary of recent efforts," Medicinal Research Reviews, vol. 37, no. 4, pp. 860-906, 2016.

[3] H. L. Paulson, V. G. Shakkottai, H. B. Clark, and H. T. Orr, "Polyglutamine spinocerebellar ataxias - from genes to potential treatments," Nature Reviews. Neuroscience, vol. 18, no. 10, pp. 613-626, 2017.

[4] I. Paradisi, V. Ikonomu, and S. Arias, "Huntington disease-like 2 (HDL2) in Venezuela: frequency and ethnic origin," Journal of Human Genetics, vol. 58, no. 1, pp. 3-6, 2013.

[5] C. Wang, Z. Chen, F. Yang et al., "Analysis of the GGGGCC repeat expansions of the C9orf72 gene in SCA3/MJD patients from China," PLoS One, vol. 10, no. 6, article e0130336, 2015.

[6] X. Li, H. Liu, P. L. Fischhaber, and T. S. Tang, "Toward therapeutic targets for SCA3: insight into the role of Machado-Joseph disease protein ataxin-3 in misfolded proteins clearance," Progress in Neurobiology, vol. 132, pp. 34-58, 2015.

[7] H. Peng, C. Wang, Z. Chen et al., "The APOE $\varepsilon 2$ allele may decrease the age at onset in patients with spinocerebellar ataxia type 3 or Machado-Joseph disease from the Chinese Han Population," Neurobiology of Aging, vol. 35, no. 9, pp. 2179.e152179.e18, 2014.

[8] Z. Chen, P. Wang, C. Wang et al., "Updated frequency analysis of spinocerebellar ataxia in China," Brain, vol. 141, no. 4, article e22, 2018.

[9] C. Wang, H. Peng, J. Li et al., "Alteration of methylation status in the ATXN3 gene promoter region is linked to the SCA3/ MJD," Neurobiology of Aging, vol. 53, pp. 192.e5-192.e10, 2017.

[10] Z. Chen, C. Zheng, Z. Long et al., "(CAG)n loci as genetic modifiers of age-at-onset in patients with Machado-Joseph disease from mainland China," Brain, vol. 139, no. 8, article e41, 2016. 
[11] C. Costa Mdo and H. L. Paulson, "Toward understanding Machado-Joseph disease," Progress in Neurobiology, vol. 97, no. 2, pp. 239-257, 2012.

[12] K. Seidel, S. Siswanto, M. Fredrich et al., "Polyglutamine aggregation in Huntington's disease and spinocerebellar ataxia type 3: similar mechanisms in aggregate formation," Neuropathology and Applied Neurobiology, vol. 42, no. 2, pp. 153-166, 2016.

[13] K. Rajamani, J. W. Liu, C. H. Wu et al., "N-Butylidenephthalide exhibits protection against neurotoxicity through regulation of tryptophan 2, 3 dioxygenase in spinocerebellar ataxia type 3," Neuropharmacology, vol. 117, pp. 434-446, 2017.

[14] M. Tada, M. Nishizawa, and O. Onodera, "Roles of inositol 1,4,5-trisphosphate receptors in spinocerebellar ataxias," Neurochemistry International, vol. 94, pp. 1-8, 2016.

[15] X. Chen, J. Wu, Y. Luo et al., "Expanded polyglutaminebinding peptoid as a novel therapeutic agent for treatment of Huntington's disease," Chemistry \& Biology, vol. 18, no. 9, pp. 1113-1125, 2011.

[16] A. H. Chou, Y. L. Chen, C. C. Chiu et al., "T1-11 and JMF1907 ameliorate polyglutamine-expanded ataxin-3induced neurodegeneration, transcriptional dysregulation and ataxic symptom in the SCA3 transgenic mouse," Neuropharmacology, vol. 99, pp. 308-317, 2015.

[17] S. Gunawardena, L.-S. Her, R. G. Brusch et al., "Disruption of axonal transport by loss of huntingtin or expression of pathogenic polyQ proteins in Drosophila," Neuron, vol. 40, no. 1, pp. 25-40, 2003.

[18] B. Malik, N. Nirmalananthan, L. G. Bilsland et al., "Absence of disturbed axonal transport in spinal and bulbar muscular atrophy," Human Molecular Genetics, vol. 20, no. 9, pp. 17761786, 2011.

[19] J. C. Chang, S. L. Wu, F. Hoel et al., "Far-infrared radiation protects viability in a cell model of spinocerebellar ataxia by preventing polyQ protein accumulation and improving mitochondrial function," Scientific Reports, vol. 6, no. 1, article 30436, 2016

[20] J. Y. Hsu, Y. L. Jhang, P. H. Cheng et al., "The truncated Cterminal fragment of mutant ATXN3 disrupts mitochondria dynamics in spinocerebellar ataxia type 3 models," Frontiers in Molecular Neuroscience, vol. 10, p. 196, 2017.

[21] F. P. Marchese, I. Raimondi, and M. Huarte, "The multidimensional mechanisms of long noncoding RNA function," Genome Biology, vol. 18, no. 1, p. 206, 2017.

[22] J. Beermann, M. T. Piccoli, J. Viereck, and T. Thum, "Noncoding RNAs in development and disease: background, mechanisms, and therapeutic approaches," Physiological Reviews, vol. 96, no. 4, pp. 1297-1325, 2016.

[23] F. Kopp and J. T. Mendell, "Functional classification and experimental dissection of long noncoding RNAs," Cell, vol. 172, no. 3, pp. 393-407, 2018.

[24] M. L. Moseley, T. Zu, Y. Ikeda et al., "Bidirectional expression of CUG and CAG expansion transcripts and intranuclear polyglutamine inclusions in spinocerebellar ataxia type 8 ," Nature Genetics, vol. 38, no. 7, pp. 758-769, 2006.

[25] J. S. Sunwoo, S. T. Lee, W. Im et al., "Altered expression of the long noncoding RNA NEAT1 in Huntington's disease," Molecular Neurobiology, vol. 54, no. 2, pp. 1577-1586, 2017.
[26] C. K. Cemal, C. Huxley, and S. Chamberlain, "Insertion of expanded CAG trinucleotide repeat motifs into a yeast artificial chromosome containing the human Machado-Joseph disease gene," Gene, vol. 236, no. 1, pp. 53-61, 1999.

[27] C. K. Cemal, C. J. Carroll, L. Lawrence et al., "YAC transgenic mice carrying pathological alleles of the MJD1 locus exhibit a mild and slowly progressive cerebellar deficit," Human Molecular Genetics, vol. 11, no. 9, pp. 1075-1094, 2002.

[28] D. Parkhomchuk, T. Borodina, V. Amstislavskiy et al., "Transcriptome analysis by strand-specific sequencing of complementary DNA," Nucleic Acids Research, vol. 37, no. 18, article e123, 2009.

[29] F. Mori, M. Nishie, Y. S. Piao et al., "Accumulation of NEDD8 in neuronal and glial inclusions of neurodegenerative disorders," Neuropathology and Applied Neurobiology, vol. 31, no. 1, pp. 53-61, 2005.

[30] T. Mueller, P. Breuer, I. Schmitt, J. Walter, B. O. Evert, and U. Wüllner, "CK2-dependent phosphorylation determines cellular localization and stability of ataxin-3," Human Molecular Genetics, vol. 18, no. 17, pp. 3334-3343, 2009. 


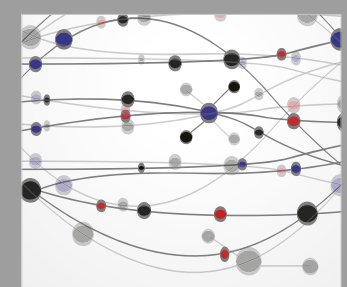

The Scientific World Journal
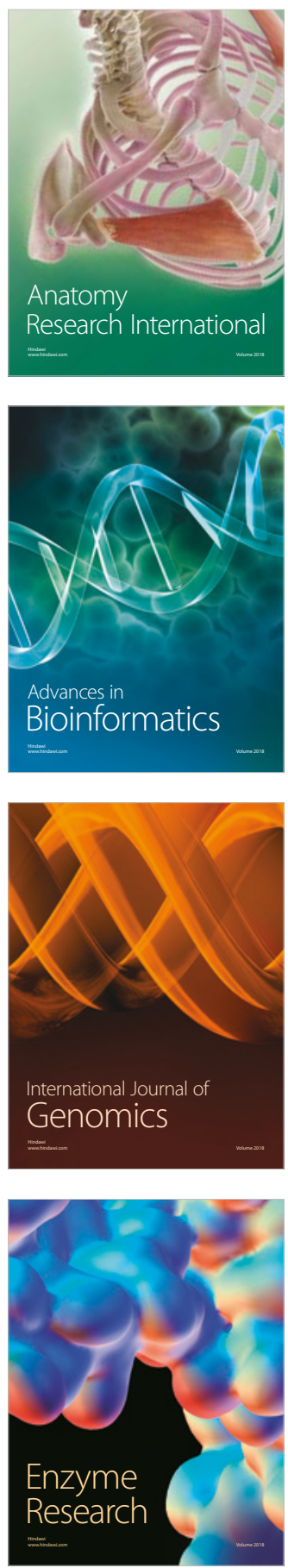
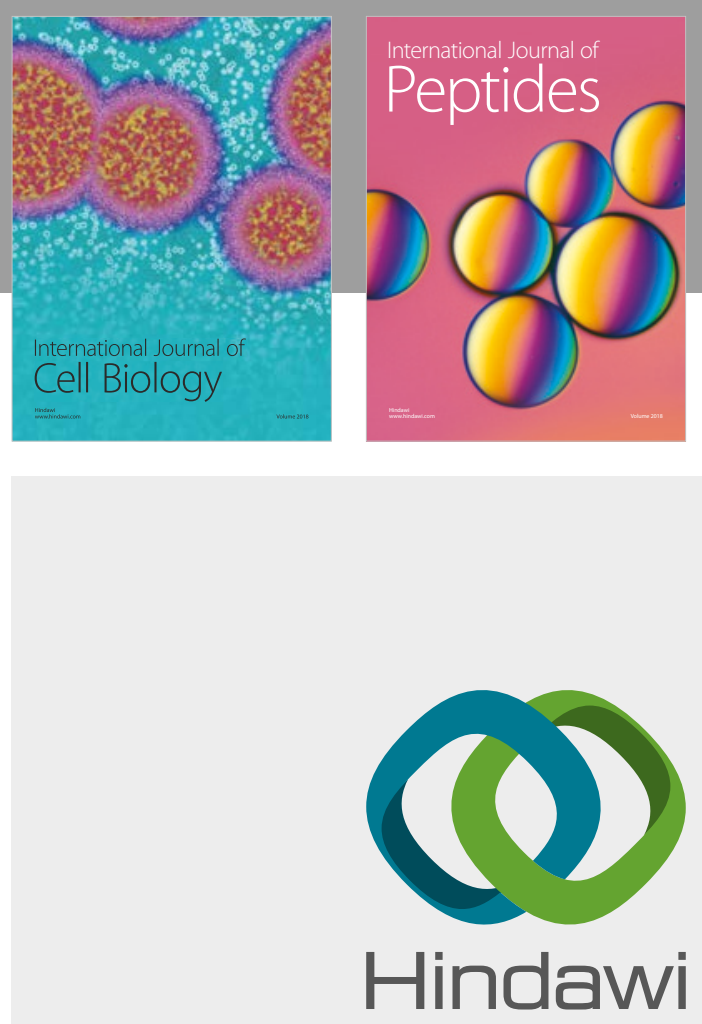

Submit your manuscripts at

www.hindawi.com
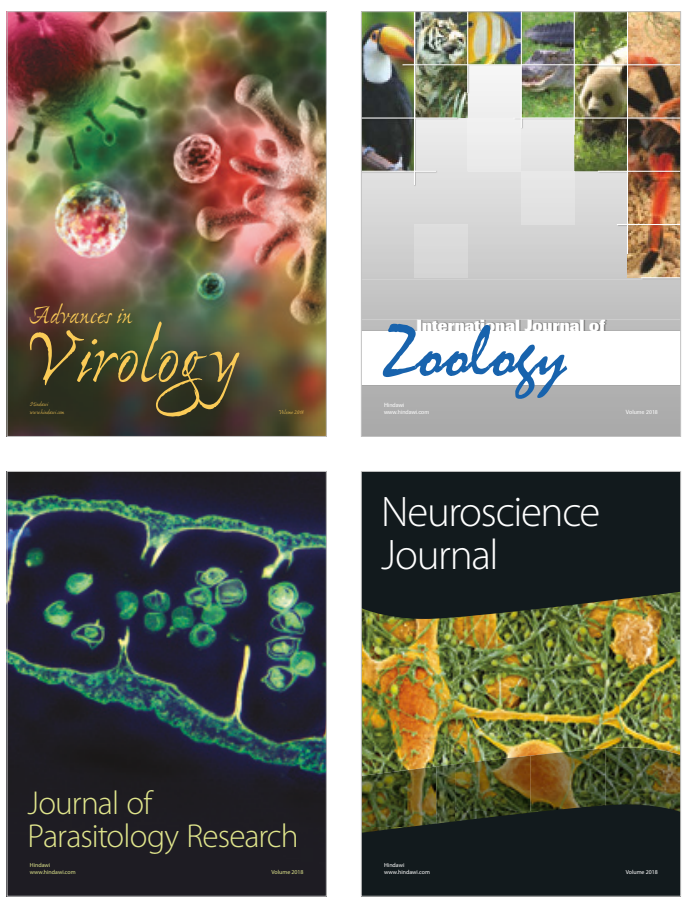
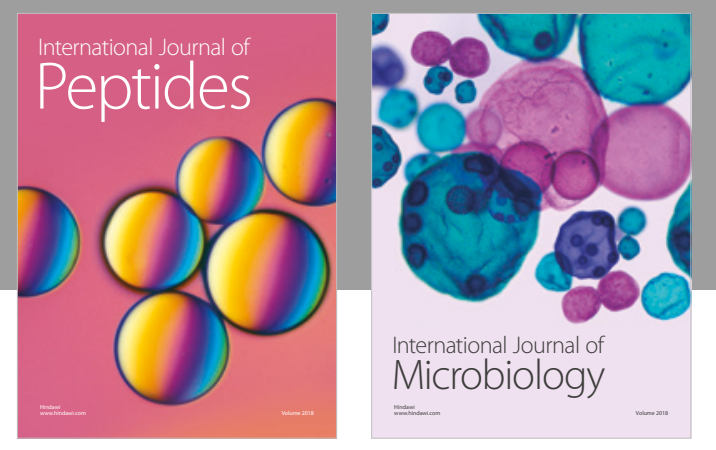

nternational Journal of Microbiology
Journal of
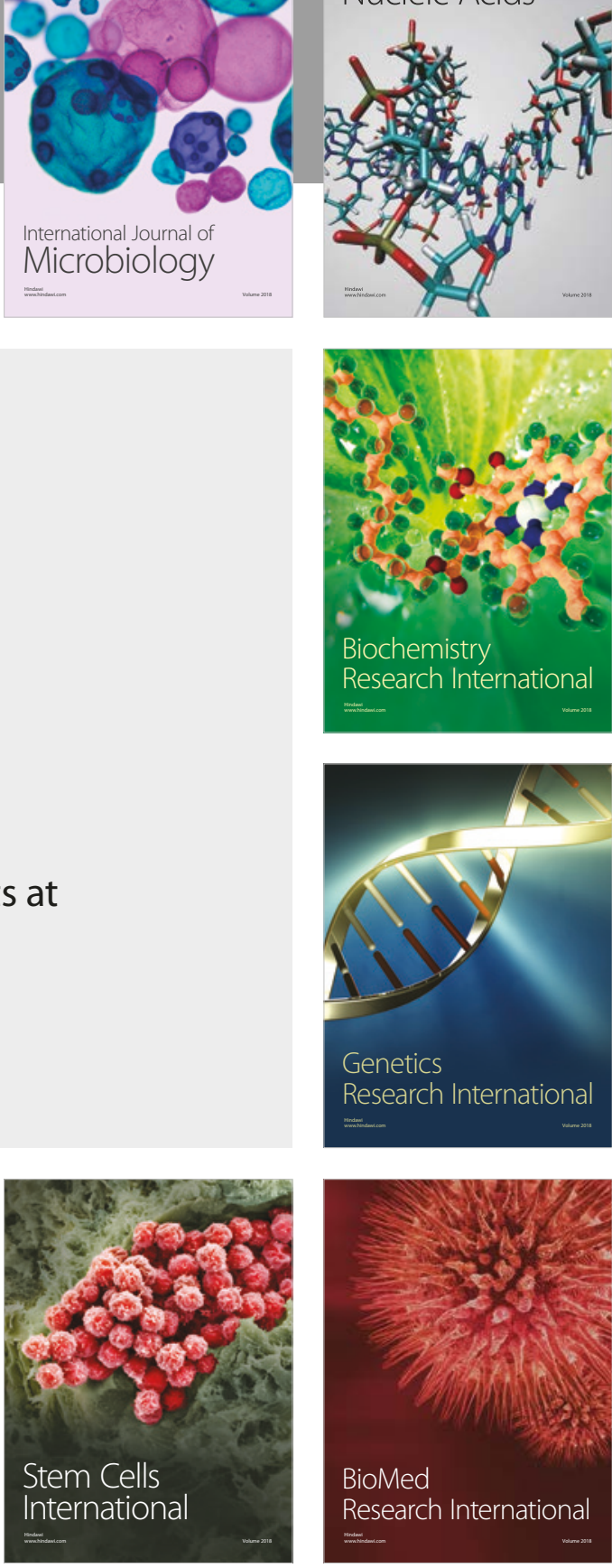
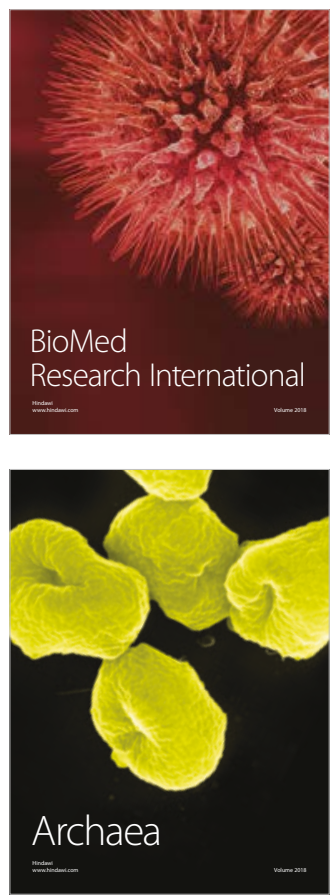